\title{
Ion Beam Induced Artifacts in Lead Based Chalcogenides
}

Xiaomi Zhang ${ }^{1}$, Shiqiang Hao ${ }^{1}$, Gangjian Tan $^{2}$, Xiaobing Hu${ }^{1,3}$, Eric W. Roth ${ }^{3}$, Mercouri G. Kanatzidis ${ }^{2}$, Chris Wolverton ${ }^{1}$ and Vinayak P. Dravid ${ }^{1,3^{*}}$

1. Department of Materials Science and Engineering, Northwestern University, Evanston, IL, USA.

2. Department of Chemistry, Northwestern University, Evanston, IL, USA.

3. NUANCE Center, Northwestern University, Evanston, IL, USA.

* Corresponding author: v-dravid@northwestern.edu

Metal chalcogenides have shown great potential in applications such as solar cell, topological insulators, thermoelectric materials along with others. It has been well demonstrated that microstructure can alter intrinsic properties and performance of metal chalcogenides.[1-3] The structure-property-performance relationships can be investigated at atomic scale with scanning transmission and transmission electron microscopy. Nevertheless, careful specimen preparation is paramount for accurate analyses and interpretations. Different artifacts can be induced during specimen preparation depending on the specimen preparation method being used, which can make the interpretation of TEM images difficult. Discerning between intrinsic material features and potential artifacts is vital for determining the structure-propertyperformance relationship in metal chalcogenides.

Here, we characterized and compared the microstructure of ingot stoichiometric PbTe TEM specimen prepared with five different specimen preparation methods (as shown in Figure 1). Intriguingly, we revealed that specimen preparation methods that involves high energy $(>1 \mathrm{keV})$ ion-specimen interaction will introduce artifacts that appear as orthogonal dark line segments. These artifacts are approximately 3$10 \mathrm{~nm}$ in length and about 2-5 lattice fringes in width as shown in the inset figure of Figure 1 (d). The long sides are parallel to $<001>$ crystal orientations.

We demonstrated that with low energy $(300 \mathrm{eV})$ Ar ion cleaning, the artifacts observed can be significantly reduced. The fact that these artifacts can be eliminated implies that they are surface damage induced by high energy ion bombardment. The appearance of these artifacts under TEM indicates they can be point defect clusters, dislocations or nanoscale precipitates. A series of experiments were performed to determine the structural and chemical nature of these artifacts. In-situ heating of ingot stoichiometric $\mathrm{PbTe}$ specimen prepared by high energy ion milling were performed, the orthogonal features got eliminated at $220{ }^{\circ} \mathrm{C}$ inside the TEM. Since the heating temperature is well below the eutectic temperature of the $\mathrm{Pb}-$ Te binary phase diagram, if the orthogonal features were nanoscale precipitates, nucleation and coarsening should be observed. Instead, we observed the elimination of these orthogonal features, indicating these features should be either point defect clusters or dislocations. Aberration corrected scanning transmission electron microscopy (Figure 2), together with first principles calculation, disclosed that the orthogonal artifacts should be $\mathrm{Pb}$ self-interstitial atom clusters.

In this presentation, we will explain the reason of different observed microstructure in specimens prepared with different methods, identify the specimen preparation induced artifacts, disclose the chemical nature of these artifacts and demonstrate proper specimen preparation protocol. Understanding the intrinsic microstructure is very essential in correctly attribute the interesting properties and performances of metal chalcogenide materials. TEM specimen preparation needs to be performed cautiously to maintain materials real microstructure and avoid artifacts [4]. 
References:

[1] G Tan, LD Zhao and MG Kanatzidis, Chem Rev 116 (2016), p. 12123.

[2] Y Zhao and C Burda, Energy \& Environmental Science 5 (2012), p. 5564.

[3] Y Liu et al., Nature Physics 10 (2014), p. 294.

[4] This material is based upon work supported by U. S. Department of Energy, Office of Science, and Office of Basic Energy Sciences under Award Number DE-SC-0014520. This work made use of the EPIC facility of Northwestern University's NUANCE Center, which has received support from the Soft and Hybrid Nanotechnology Experimental (SHyNE) Resource (NSF ECCS-1542205); the MRSEC program (NSF DMR-1720139) at the Materials Research Center; the International Institute for Nanotechnology (IIN); the Keck Foundation; and the State of Illinois, through the IIN.
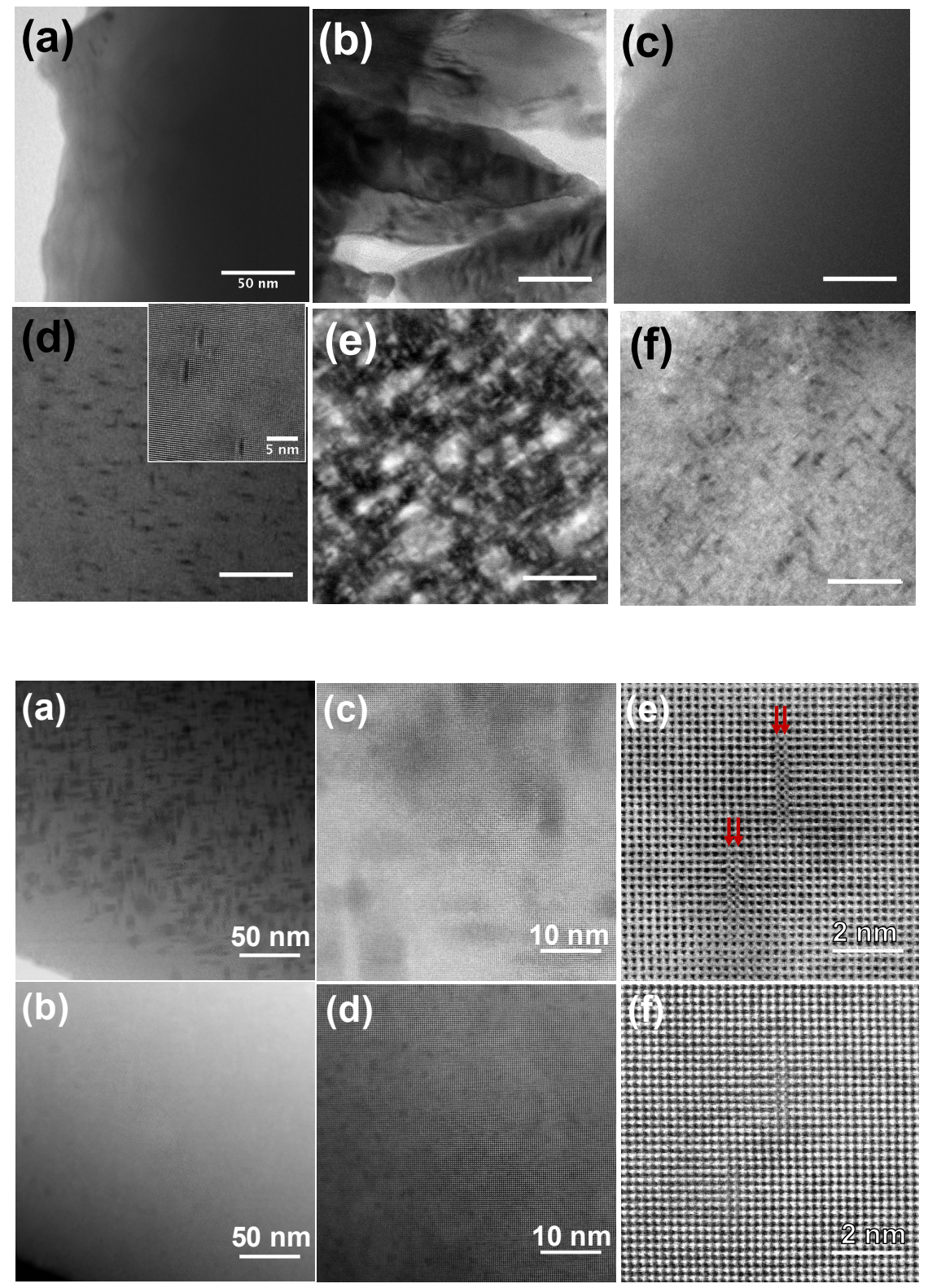

Figure 1. Transmission electron microscopy of ingot PbTe specimens prepared with different methods. (a)(d) are conventional TEM image of ingot $\mathrm{PbTe}$ prepared by crushing, ultramicrotomy, twin jet electropolishing, and ion milling, respectively. (e) is the ADF STEM image of ingot $\mathrm{PbTe}$ prepared by FIB thinning. (f) is a conventional TEM image of the same TEM sample used in (c) but then ion milled for 25 minutes. The upper right inset image in (d) is a high resolution image of typical orthogonal nanoscale features observed in ion milled specimens. Scale bars in (a)-(f) represent $50 \mathrm{~nm}$.

Figure 2. STEM of ingot $\mathrm{PbTe}$ specimen prepared by ion milling. (a) STEM BF and (b) STEM HAADF low magnification images simultaneously obtained at same region of interest. (a) depicts large number density of orthogonal features while (b) shows very minimal contrast. (c) STEM BF and (d) STEM HAADF high magnification image simultaneously obtained at same region of interest. (c) depicts orthogonal features while (d) shows large number density, homogeneously distributed dark clusters. (e) STEM BF and (f) STEM HAADF high-resolution images obtained simultaneously at the same region of interest. Self interstitial clusters can be observed (indicated by red arrows). 